OPEN ACCESS

Edited by:

Joseph M. Bliss,

Women \& Infants Hospital

of Rhode Island, USA

Reviewed by:

Katie Louise Flanagan,

Monash University, Australia

Juliana Cassataro,

National Scientific and Technical

Research Council, Argentina

Patricia Talamás-Rohana,

CINVESTAV, Mexico

${ }^{*}$ Correspondence:

Ofer Levy

ofer.levy@childrens.harvard.edu

Specialty section:

This article was submitted to Microbial Immunology, a section of the journal

Frontiers in Immunology

Received: 10 October 2016 Accepted: 22 November 2016

Published: 08 December 2016

Citation:

Dowling DJ, Sanders H, Cheng WK Joshi S, Brightman S, Bergelson I,

Pietrasanta C, van Haren SD, van Amsterdam S, Fernandez J, van den Dobbelsteen GPJM and Levy O (2016) A Meningococcal Outer Membrane Vesicle Vaccine Incorporating Genetically Attenuated Endotoxin Dissociates Inflammation from Immunogenicity. Front. Immunol. 7:562. doi: 10.3389/fimmu.2016.00562

\section{A Meningococcal Outer Membrane Vesicle Vaccine Incorporating Genetically Attenuated Endotoxin Dissociates Inflammation from Immunogenicity}

David J. Dowling ${ }^{1,2}$, Holly Sanders ${ }^{3}$, Wing Ki Cheng ${ }^{1,2,4}$, Sweta Joshi',4, Spencer Brightman ${ }^{1,4}$, Ilana Bergelson', Carlo Pietrasanta ${ }^{1,2,4,5}$, Simon D. van Haren ${ }^{1,2,4}$, Sandra van Amsterdam ${ }^{3}$, Jeffrey Fernandez ${ }^{6}$, Germie P. J. M. van den Dobbelsteen ${ }^{3}$ and Ofer Levy ${ }^{1,2,4 *}$

\footnotetext{
${ }^{1}$ Department of Medicine, Division of Infectious Diseases, Boston Children's Hospital, Boston, MA, USA, ${ }^{2}$ Harvard Medical School, Boston, MA, USA, ${ }^{3}$ Janssen Vaccines and Prevention B.V., Leiden, Netherlands, ${ }^{4}$ Precision Vaccine Program, Division of Infectious Diseases, Boston Children's Hospital, Boston, MA, USA, ${ }^{5}$ Neonatal Intensive Care Unit, Department of Clinical Sciences and Community Health, Fondazione IRCCS Ca' Granda Ospedale Maggiore Policlinico, University of Milan, Milan, Italy, ${ }^{6}$ Janssen Research and Development, LLC, Spring House, PA, USA
}

Background: Group B Neisseria meningitidis, an endotoxin-producing Gram-negative bacterium, causes the highest incidence of group B meningococcus (MenB) disease in the first year of life. The Bexsero vaccine is indicated in Europe from 8 weeks of age. Endotoxin components of outer membrane vesicles (OMVs) or soluble lipopolysaccharide (LPS) represent a potential source of inflammation and residual reactogenicity. The purpose of this study was to compare novel candidate MenB vaccine formulations with licensed vaccines, including Bexsero, using age-specific human in vitro culture systems.

Methods: OMVs from wild type- and inactivated IpxL1 gene mutant-N. meningitidis strains were characterized in human neonatal and adult in vitro whole blood assays and dendritic cell (DC) arrays. OMVs were benchmarked against licensed vaccines, including Bexsero and whole cell pertussis formulations, with respect to Th-polarizing cytokine and prostaglandin E2 production, as well as cell surface activation markers (HLA-DR, CD86, and CCR7). OMV immunogenicity was assessed in mice.

Results: $\Delta / p \times L /$ native OMVs (nOMVs) demonstrated significantly less cytokine induction in human blood and DCs than Bexsero and most of the other pediatric vaccines (e.g., PedvaxHib, EasyFive, and bacillus Calmette-Guérin) tested. Despite a much lower inflammatory profile in vitro than Bexsero, $\Delta / p \times L /$ nOMVs still had moderate DC maturing ability and induced robust anti- $N$. meningitidis antibody responses after murine immunization.

Conclusion: A meningococcal vaccine comprised of attenuated LPS-based OMVs with a limited inflammatory profile in vitro induces robust antigen-specific immunogenicity in vivo.

Keywords: group B meningococci, outer membrane vesicles, vaccine, newborn, dendritic cells 


\section{INTRODUCTION}

Human newborns and infants suffer a high frequency of infection compared to older children and adults (1), in part due to distinct early life immunity with impaired host defense (2). Early life immunization is desirable, but vaccine-induced responses of newborns and young infants demonstrate slow initiation, low immunogenicity, and reduced persistence of functional antibodies and cell-mediated responses (2). Although the majority of global immunization schedules are focused on the pediatric age group, development of early life vaccines has been hampered by this distinct immunity and an ad hoc approach to developing vaccines, relying on adult-derived results that may inaccurately predict infant responses (3).

Neisseria meningitidis (meningococcus) is a Gram-negative, endotoxin-producing organism that is a normal commensal of the human nasopharynx and is an important cause of invasive bacterial infection in children worldwide (4). Recent immunization programs with capsular-polysaccharide vaccines have dramatically reduced the incidence of serogroup $\mathrm{C}$ and $\mathrm{A}$ meningococcal disease in North America, Europe, and Africa (5). However, meningitis and septicemia caused by serogroup B meningococci remain a major health concern in young children, as similar capsule-based vaccines cannot be developed against this serogroup (6). Outer membrane vesicle (OMV)-based vaccines have historically been used, with some success, to control outbreaks of disease caused by serogroup B meningococci. OMVs are produced by the blebbing of membranes of clinicalderived live Gram-negative bacteria during in vitro growth and are useful vaccine components, as immuno-stimulatory membrane components [lipids, proteins, lipopolysaccharide (LPS), etc.] from meningococci are represented (7). As OMV yield from culture alone is too low for vaccine production, detergent extraction is used to force vesiculation and increase yield and has the added advantage of reducing LPS content to prevent overt reactogenicity. Novartis' 4-component aluminum hydroxide (Alum)-adjuvanted group B meningococcus (MenB) vaccine (4CMenB, Trade name: Bexsero) has been licensed by both the European Medicines Agency and the U.S. Food and Drug Agency and comprised OMVs and three recombinant immunogenic $N$. meningitidis proteins identified by reverse vaccinology (8). Another meningococcal serogroup B vaccine, Trumenba, contains two recombinant proteins with no OMV component, is approved in the U.S. for use in individuals 10 through 25 years of age, but lacks the potentially broader antigen repertoire inherent to OMVs.

The currently licensed meningococcal vaccines have the potential to reduce mortality and morbidity associated with MenB infections, but do have some limitations. Although detergent extraction of OMVs removes the majority of the LPS, the remaining endotoxin, particularly the soluble LPS, may still result in residual reactogenicity, necessitating the use of the Alum to ameliorate excess toxicity (9). Even so, immunization with the OMV-containing Bexsero may correlate with rates of reactogenicity (e.g., fever $\geq 38^{\circ} \mathrm{C}$, tenderness at injection site) as common as $10 \%$ in infants less than 1 years of age (10), and severe reactogenicity is reported in some cases (11). Bexsero may also enhance reactogenicity when given together with other vaccines (12), prompting some primary care physicians to prescribe anti-inflammatory agents (13) to prevent potential reactions. Furthermore, as conventional assays for preclinical and release testing of vaccines, such as the rabbit pyrogenicity test and Limulus amebocyte lysate (LAL) assay, were developed for vaccines containing no or negligible amounts of endotoxin, predicting the reactogenicity of LPS-containing OMV vaccines is difficult and requires more sophisticated models (14).

To overcome the potential reactogenicity of wild-type (WT) OMV-based vaccines while maintaining the inherent adjuvant activity of vesicles, a vaccine has previously been developed using a $N$. meningitidis strain with genetically attenuated endotoxin (15). This enabled the use of detergent-free manufacturing processes to produce native OMVs (nOMVs) (16). To assess the potential of such OMV vaccines, we reasoned that it would be important to better understand their interactions with human leukocytes, including dendritic cells (DCs). DCs are professional antigen-presenting cells (APCs) that play a vital role in shaping adaptive immunity. DC maturation begins when endogenous or exogenous danger molecules are recognized by pattern recognition receptors [e.g., Toll-like receptors (TLRs)] triggering upregulation of costimulatory molecules and production of immune polarizing cytokines (17). Of note, in addition to interactions via TLR4, N. meningitidis LPS also directly interacts with DCs through the C-type lectin receptor DC-SIGN and modulates their function (18).

In this study, we benchmarked novel candidate MenB vaccine formulations against licensed vaccines, including Bexsero, using physiologically relevant human neonatal and adult whole blood (WBA) and monocyte-derived dendritic cell (MoDC) in vitro culture systems, both of which employ age-specific autologous plasma, a rich source of age-specific immunomodulatory molecules $(2,19)$. We assessed vaccine-induced production of Th-polarizing cytokines, upregulation of surface activation markers (20), and production of potential vaccine reactogenicity biomarkers such as IL-1 $\beta$ and prostaglandin E2 ( $\left.\mathrm{PGE}_{2}\right)(3$, $21,22)$. We found that a meningococcal vaccine comprised of attenuated LPS-based OMVs with a relatively low inflammatory profile toward human leukocytes in vitro induced robust antigen-specific immunogenicity in vivo. Our observations, may inform translational development of MenB vaccines in high-risk populations, such as newborns and infants.

\section{MATERIALS AND METHODS}

\section{Ethics Statement}

Peripheral blood samples were collected after written informed consent from healthy adult volunteers with approval from the Ethics Committee of Boston Children's Hospital, Boston, MA, USA (X07-05-0223). Non-identifiable cord blood samples were collected immediately after elective cesarean section delivery (epidural anesthesia) of the placenta. Births to HIV-positive or febrile mothers were excluded. Human experimentation guidelines of the U.S. Department of Health and Human Services, the Brigham and Women's Hospital, Beth Israel Medical Center, and Boston Children's Hospital were observed, with approval 
from the Ethics Committee of The Brigham and Women's Hospital, Boston, MA, USA (protocol number 2000-P-000117) and Beth Israel Deaconess Medical Center Boston, MA, USA (2011P-000118). Mice were obtained from Envigo (Indianapolis, IN, USA), and studies were approved by the Janssen Research and Development, LLC Institutional Animal Care and Use Committee.

\section{Human Blood Processing and In Vitro Stimulation}

Human blood was anti-coagulated with $20 \mathrm{U} / \mathrm{ml}$ pyrogen-free sodium heparin (American Pharmaceutical Partners, Inc., Schaumberg, IL, USA). All blood products were kept at room temperature and processed within $4 \mathrm{~h}$ from collection. Whole blood (WB) was mixed 1:1 with pre-warmed $\left(37^{\circ} \mathrm{C}\right) \mathrm{RPMI} 1640$ medium (Invitrogen, Carlsbad, CA, USA) and $180 \mu$ plated in a 96-well U-bottom plate (Becton Dickinson, Franklin Lakes, NJ, USA) containing $20 \mu \mathrm{l}$ of $10 \times$ freshly prepared treatment, as described previously (23).

\section{Human MoDCs}

Blood was layered onto Ficoll-Hypaque gradient (GE Healthcare, Waukesha, WI, USA) to collect cord blood mononuclear cells (CBMCs) or peripheral blood mononuclear cells (PBMCs). Monocytes were isolated by positive CD14 selection with magnetic microbeads (Miltenyi Biotec, Auburn, CA, USA). Preparations were routinely $>95 \%$ pure as assessed by flow cytometry (23-26). Monocytes were cultured in tissue culture dishes at $0.4 \times 10^{6}$ cells/ $\mathrm{ml}$ in RPMI media containing fresh $10 \%$ autologous platelet-poor plasma, supplemented with recombinant human (rh) IL-4 (50 ng/ $\mathrm{ml}$ ) and rh granulocyte-macrophage colony-stimulating factor (rhGM-CSF) (100 ng/ml) (R\&D Systems, Minneapolis, MN, USA) with one replenishment of fresh media/cytokines at day 3. After 5-6 days, immature MoDCs were handled, as described previously $(23,25)$.

\section{Vaccines, TLR Agonists, and Multi-Analyte Assays}

Licensed vaccines were commercially obtained. Ultrapure LPS (Salmonella minnesota R595, List Biological Laboratories, Campbell, CA, USA) was used at $100 \mathrm{ng} / \mathrm{ml}$. Supernatants were assayed by ELISA for TNF (BD Biosciences, San Jose, CA, USA), IL-1 $\beta$ (eBiosciences, San Diego, CA, USA) and by competitive monoclonal enzyme immunoassay (EIA) for $\mathrm{PGE}_{2}$ (Cayman Chemical, Ann Arbor, MI, USA). Additionally, assay supernatants were analyzed by magnetic bead multiplex cytokine/ chemokine assay (Millipore, Billerica, MA, USA) and analyzed on the Luminex ${ }^{\circledR} 100 / 200^{\mathrm{TM}}$ System employing XPOTENT $^{\circledR}$ software (Luminex, Austin, TX, USA) and Millipore Milliplex Analyst (version 3.5.5.0). $\mathrm{PGE}_{2}$ concentrations were determined using the analysis tool at www.myassays.com.

\section{Flow Cytometry}

Monocyte-derived dendritic cells were treated with blocking agent for $10 \mathrm{~min}$ (Sigma-Aldrich), transferred to staining buffer
[1× PBS, $0.5 \%(\mathrm{v} / \mathrm{v})$ human serum albumin] and stained for $30 \mathrm{~min}$ at $4^{\circ} \mathrm{C}$ in the dark $\left(1 \times 10^{5}\right.$ cells/staining $)$ with fluorophore-labeled antibodies [CD80/Fluorescein isothiocyanate/ Clone L307.4, CD86/phycoerythrin/Clone 2331, CCR7/V450/ Clone 150503, and HLA-DR/PerCP-Cy5.5/Clone L243 (BD Biosciences)]. Cells were then centrifuged $(500 \times g, 10 \mathrm{~min})$, washed, fixed [ $1 \%(\mathrm{v} / \mathrm{v})$ paraformaldehyde], and acquired using a LSRII flow cytometer employing FACSDiva software (BD Biosciences). Data were analyzed using the FlowJo (Tree Star, Inc., Ashland, OR, USA). Typically, 10,000 events per sample were acquired.

\section{Production of OMVs and Characterization}

Outer membrane vesicles were provided by Janssen Vaccines and Prevention B.V. Briefly, OMVs were produced from $N$. meningitidis $\mathrm{H} 44 / 76$ (B:P1.7,16;F3-3) WT or derivatives. nOMVs were produced from a $H 44 / 76 \Delta \mathrm{R} \Delta \mathrm{L}(15,16,27)$, resulting in a pentaacylated meningococcal LPS from the $l p x L 1$ mutant $N$. meningitidis strain, lacking the secondary C12:0 acyl chain at the $2^{\prime}$-position of the lipid A. Detergent-extracted OMVs were produced from the WT strain or the $\Delta \mathrm{R} \Delta \mathrm{L}$ mutant (28). Adsorption to aluminum hydroxide (Sigma-Aldrich) was performed, as described elsewhere (29). Before use, OMVs were diluted in $10 \mathrm{mM}$ Tris $\mathrm{pH} 7.4 / 3 \%(\mathrm{w} / \mathrm{v})$ sucrose to $50 \mu \mathrm{g}$ total protein $/ \mathrm{ml}$. OMV size was determined by dynamic light scattering (DLS) using the Zetasizer nanoseries (Malvern Nano-ZS, $11 / 4532 \mathrm{~nm}$, Westborough, MA, USA).

\section{In Vivo Immunogenicity}

Adult Balb/c mice (6-8 weeks, female, 10/group) were immunized with two doses of OMVs $(2.5 \mu \mathrm{g}$ total protein/dose), two doses of Bexsero [at $2.5 \mu \mathrm{g}$ total protein/dose (equivalent to $1 / 10$ th human dose)], or buffer control, subcutaneously in the scruff of the neck. Doses were administered 4 weeks apart, with terminal bleed taken 2 weeks after the second dose. Serum bactericidal assays (SBAs) were performed, as described previously (30), against the WT H44/76 strain with sera from individual mice using baby rabbit sera (Cedarlane, Burlington, VT, USA) as an exogenous complement source. The H44/76 strain was chosen as our OMV formulations were produced from a $N$. meningitidis H44/76 strain and since H44/76 is used as an immunogenicity indicator strain for Bexsero approval. Reported titers are the reciprocal of the serum dilution giving $50 \%$ killing. If $50 \%$ killing was not achieved the sample was assigned a titer of 4 (half of the lower limit of detection).

\section{Statistical Analyses and Graphics}

Statistical significance and graphs were generated using Prism v. 5.0b (GraphPad Software, La Jolla, CA, USA) and Microsoft Excel (Microsoft Corporation, Redmond, WA, USA). For data analyzed by normalization to control values, column statistics were conducted using the two-tailed Wilcoxon signed-rank test or unpaired Mann-Whitney test as appropriate. Results were considered significant at $p<0.05$ and indicated as follows: ${ }^{*} p<0.05,{ }^{* *} p<0.01,{ }^{* * *} p<0.001,{ }^{*} p<0.05,{ }^{* *} p<0.01$,

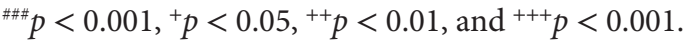




\section{RESULTS}

\section{OMVs Incorporating Genetically Attenuated Endotoxin Have Reduced Cytokine Induction Potential in Human Newborn and Adult Blood}

Wild-type OMVs were produced from $N$. meningitidis H44/76 (B:P1.7,16;F3-3), while nOMVs (Figure 1A), incorporating a genetically attenuated version of endotoxin, were produced from a $H 44 / 76 \Delta \mathrm{R} \Delta \mathrm{L}$ mutant strain $(16,27)$. All OMVs were characterized by DLS for particle size (Figure 1B; Figure S1 in Supplementary Material), with all formulations falling within a range of $80-120 \mathrm{~nm}$ in diameter. Next, we tested the ability of (1) the commercially available Bexsero vaccine, (2) detergentextracted WT OMV (WT dOMV) (i.e., a Bexsero-like OMV formulation), (3) $\Delta \mathrm{R} \Delta \mathrm{L}$ mutant nOMV strain ( $\Delta$ lpxLI nOMV) (28), and (4) a $\Delta l p x L I$ dOMV formulation (Figure 2A), to induce titration-dependent cytokine production in human neonatal and adult blood (Figures 2A,B). Non-detergent treated WT OMVs containing WT LPS were not tested as these are known

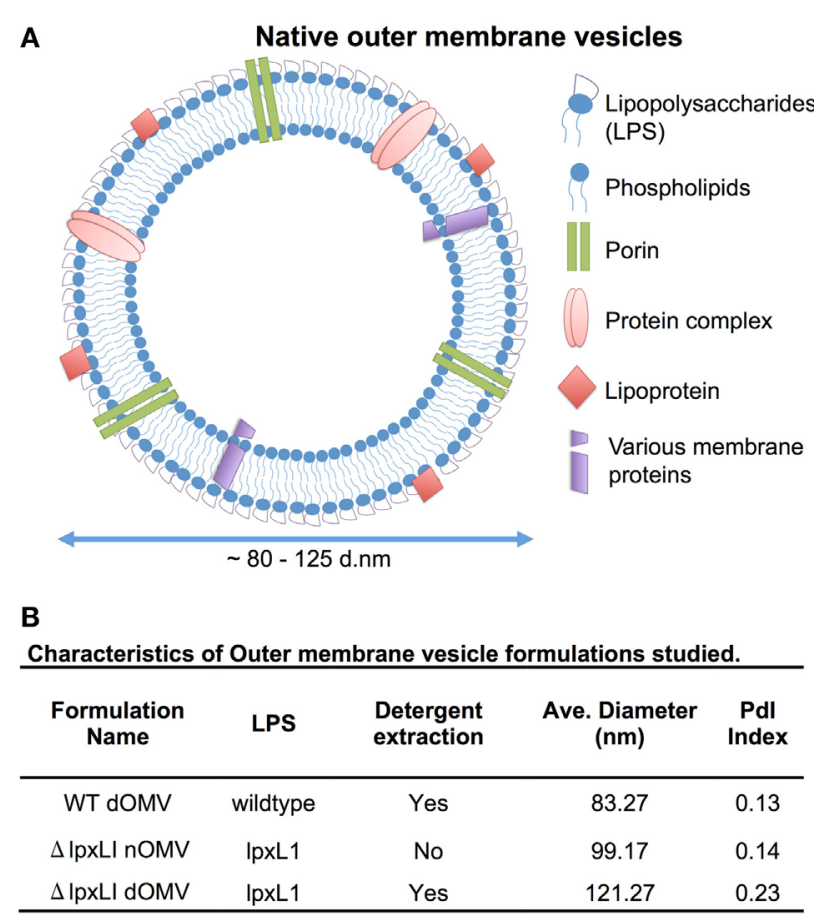

OMV: Outer membrane vesicle; LPS: Lipopolysaccharides; IpxL: Lipid A expression/biosynthesis; Pdl: Polydispersity; nm: nanometer.

FIGURE 1 | Depiction and characterization of Neisseria meningitidis outer membrane vesicles. (A) Outer membrane vesicles (OMVs) are composed of outer membrane lipids, proteins, and lipopolysaccharide and are produced by the blebbing of membranes of live Gram-negative bacteria during in vitro and in vivo growth [adapted from Sanders et al. (7)]. (B) Characterization of each OMV type used in this study, including LPS type, if detergent extraction was used, quantification of average size in nanometers and polydispersity $(\mathrm{Pdl})$ index. to be highly cytotoxic. As indicated by measurement of TNF and IL-1 $\beta$, both Bexsero and the WT dOMV robustly activated neonatal cord and adult peripheral blood in a titration-dependent manner in all concentrations tested, where levels of these two mediators were significantly increased over baseline $(p<0.001)$. The mutant OMVs also significantly induced production of TNF and IL- $1 \beta$, but only at the highest concentration in newborn blood ( $p<0.05$ ) (Figures 2A,B; Figure S2 in Supplementary Material). When compared to Bexsero at the highest equivalent volume-to-volume (v/v) dilution tested (1:10), $\Delta l p x L I$ nOMV demonstrated reduced TNF production in neonatal $(p<0.001)$ and adult $(p<0.01)$ blood. $\Delta l p x L I$ nOMV were also less effective in inducing IL- $1 \beta$ production in newborn $(p<0.05)$ and WBA $(p<0.01)$. Similar trends were observed when comparing the $\Delta l p x L I$ nOMV to WT dOMV.

We next broadened our characterization of the ability of WT $\mathrm{dOMV}, \Delta l p x L I \mathrm{dOMV}$, and $\Delta l p x L I \mathrm{nOMV}$ (at v/v 1:1000-1:10) to induce concentration-dependent cytokine production from newborn and WBA using multiplexing assays (Figures 2C,D; Figure S3 in Supplementary Material). When compared head-to-head, the $\Delta l p x L I \mathrm{dOMV}$ and $\Delta l p x L I$ nOMV induced markedly lower cytokine production profiles as compared to the WT dOMV, especially at the lowest concentration tested. The addition of Alum, the most commonly used adjuvant worldwide, and a component of the Bexsero vaccine to the OMV formulations altered the OMV (both wild type and mutant) induced innate cytokine production profiles (Figures S3 and S4 in Supplementary Material). Most notably, Alum reduced the ability of the WT dOMV to induce IFN $\gamma$ and the chemokine CXCL10 (interferon inducible 10) (Figures S5A,B in Supplementary Material).

\section{$\Delta / p x L /$ nOMV Mature Human Dendritic Cells without Bexsero-Associated Inflammatory Profile}

To further characterize the innate immune effects of OMV formulations, 96-well human MoDC-based arrays were generated after culturing CD14 selected monocytes with IL-4 and GM-CSF in the presence of autologous plasma, a rich source of age-specific soluble immunomodulatory factors (19), as described previously $(23,25)$. In response to Bexsero, a strong upregulation of CD80, HLA-DR (MHCII), and CCR7 and a modest increase in CD86 were observed in both newborn and adult DCs (Figures 3A,B). Both the $\Delta l p x L I$ nOMV and WT dOMV (with or without Alum) produced slightly reduced DC maturation profiles as compared to the Bexsero. Interestingly, the Alum-adjuvanted $\Delta l p x L I$ nOMV formulation induced a similar upregulation profile to Bexsero in adult but not newborn MoDCs. Titration-dependent induced MoDC cytokine induction (Figure 3C) supported the flow cytometry results and mostly mirrored those observed in WB. When compared head-to-head, the mutant OMV formulations induced lower cytokine production profiles as compared to the WT formulations, especially at the lowest concentration tested. Interestingly, as seen in the WBA, the addition of Alum selectively enhanced WT dOMV-induced IL-1 $\beta$ production from human DCs (Figure 3D). Moreover, the addition of Alum to the $\Delta l p x L I$ 


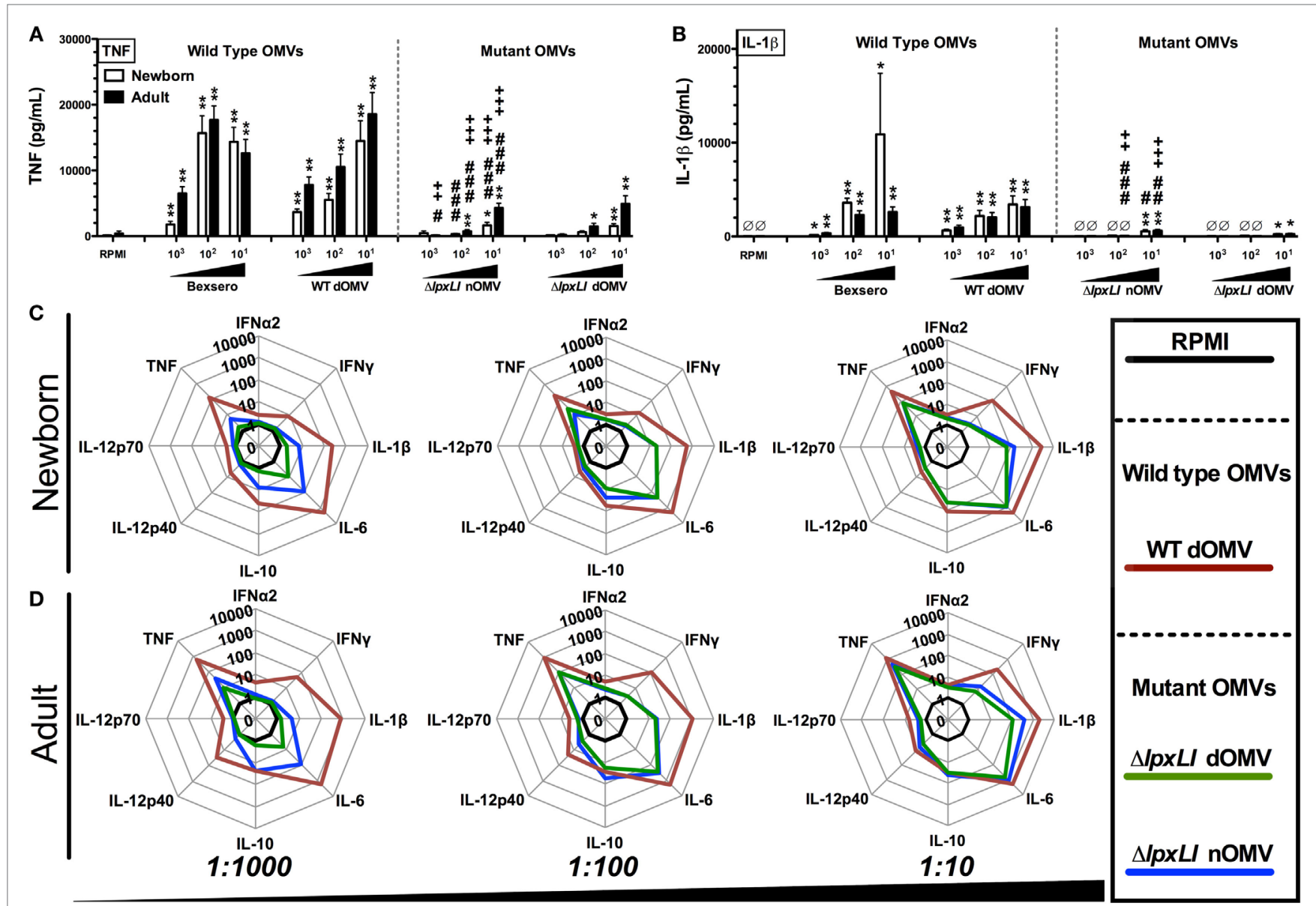

FIGURE 2 | OMVs containing genetically attenuated endotoxin demonstrate relatively low cytokine induction in human newborn and adult blood. Human neonatal and adult blood cultured in vitro for $6 \mathrm{~h}$ with buffer control (RPMl) or with increasing concentrations of wild type (WT) and mutant OMV formulations $(1: 1000-10 \mathrm{v} / \mathrm{V})$. Supernatants were collected for ELISA (A,B) and multiplex assay [(C) newborn, (D) adult]. Results represent means $\pm \mathrm{SEM}$ of $N=7$. For analyses at individual treatments (e.g., control RPMI vs. Bexero 1:10), unpaired Mann-Whitney test was applied at each concentration, and statistical significances are

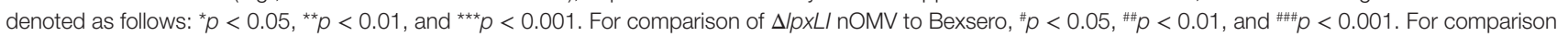
of $\Delta / p \times L /$ nOMV to WT dOMV, ${ }^{+} p<0.05,{ }^{++} p<0.01$, and ${ }^{++} p<0.001$.

nOMV formulation only induced a slight increase in IL-1 $\beta$ from newborn (Figure 3D; Figure S6A in Supplementary Material) and adult DCs (Figures 3E,F; Figure S6B in Supplementary Material) at the highest concentration tested $(1: 10 \mathrm{v} / \mathrm{v})$.

\section{$\Delta / p x L I$ nOMV Are Less Inflammatory toward Human Leukocytes than the Majority of Pediatric Vaccines}

To gain further insight into the inflammatory potential of the $\Delta l p x L I$ nOMV formulation, we next benchmarked it against a number of conventionally licensed pediatric vaccines (Table S1 in Supplementary Material) in both WB and MoDC assays. We have previously demonstrated that several of these licensed vaccines, such as the EasyFive (DTwP-HepB-Hib), induce distinct reactogenicity biomarker profiles in vitro $(31,32)$. When tested at equivalent $\mathrm{v} / \mathrm{v}$ treatment concentrations, $\Delta l p x L I$ nOMV conversely induced a lower cytokine response for most innate cytokines (Figures $\mathbf{4 A , B}$ ) and chemokines (Figure S7 in Supplementary Material) tested, grouping closer to pediatric vaccines such as PCV13 and HBV, than the more inflammatory (Alum + TLR agonist)-containing pediatric vaccines (i.e., PedvaxHIB and EasyFive).

Next, immature newborn (Figure 5A) and adult DCs (Figure 5B) were assessed for vaccine-induced production of $\mathrm{PGE}_{2}$, a molecule whose in vitro production has been correlated with reactogenicity in vivo (31-33). Of note, newborn DCs demonstrated significantly reduced $\Delta l p x L I$ nOMV-mediated $\mathrm{PGE}_{2}$ responses as compared to both Bexsero $(p<0.01)$ and WT dOMV (with Alum) $(p<0.05)$ (Figure 5A). A similar pattern was observed for adult DCs, but with significance only observed between Bexsero and $\Delta l p x L I$ nOMV $(p<0.01)$ (Figure 5B).

Newborn (Figure 5C) and adult DCs (Figure 5D) responses to $\triangle l p x L I$ nOMV were also benchmarked against conventional licensed pediatric vaccines (at 1:10 v/v, Tables S1 and S2 and Figure S8 in Supplementary Material) in the MoDC array. Here, 


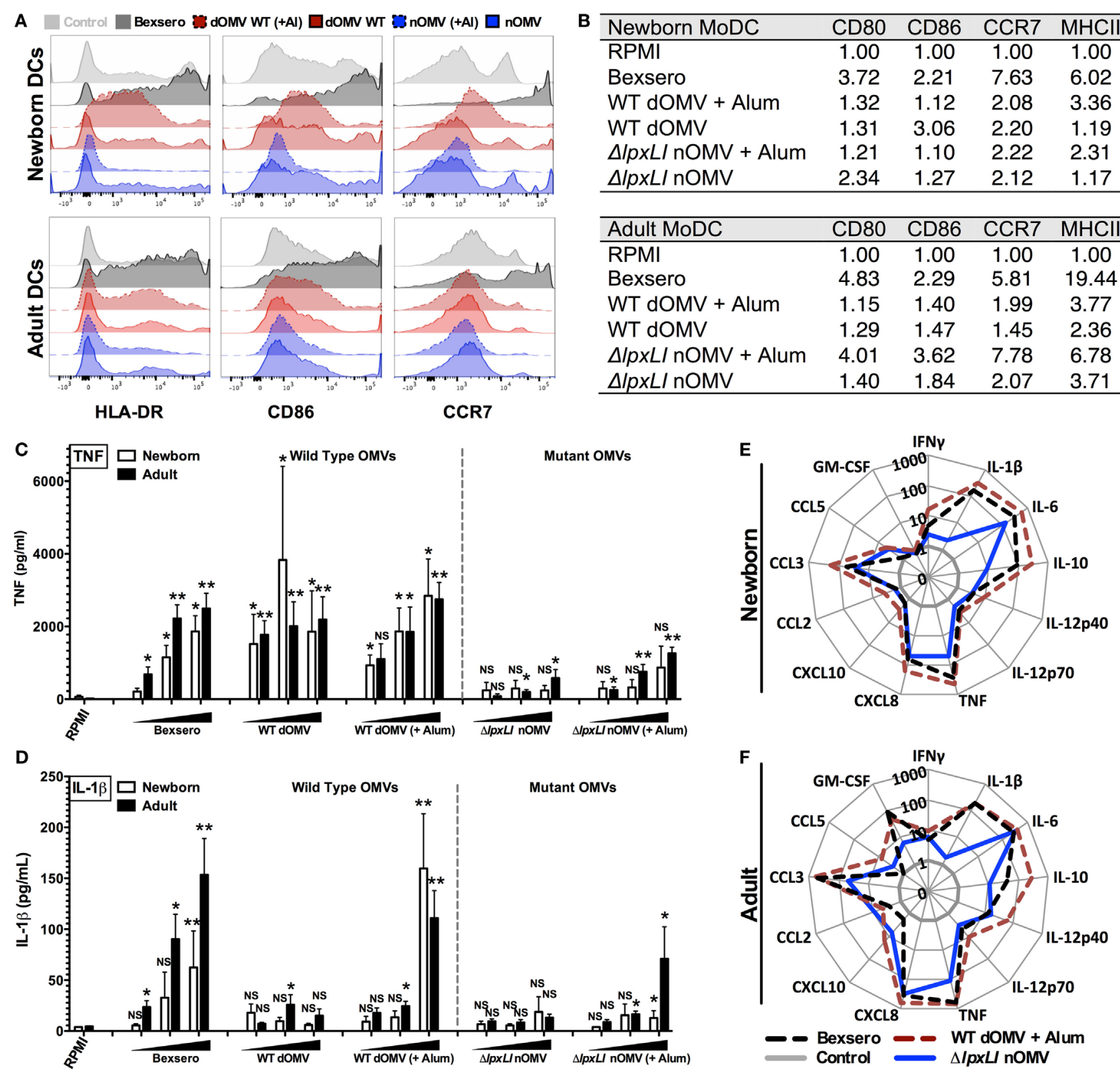

FIGURE $3 \mid \Delta / p x L I$ nOMV mature human dendritic cells without the high in vitro inflammatory profile induced by Bexsero. (A) Human newborn and adult MoDCs were cultured in $10 \%$ autologous plasma ( $/ \mathrm{V}$ ) and stimulated for $24 \mathrm{~h}$ with OMV vaccines (each at 1:10 V/V). Surface expression of costimulatory molecules and HLA was determined by flow cytometry. (B) Average MFI of newborn and adult MoDCs. (C,D) MoDCs cultured $24 \mathrm{~h}$ with increasing concentrations of OMV vaccines (each at 1:1000, 1:100, and 1:10 v/V). (E,F) Multiplex cytokine production from treated DCs. Mean $\pm \mathrm{SEM}, n=5-6$. For analyses at individual treatments (e.g., control RPMI vs. Bexsero), unpaired Mann-Whitney test was applied at each concentration, and statistical significances are denoted as follows: ${ }^{*} p<0.05$, ${ }^{* *} p<0.01$, and not significant (NS).

we focused on the correlation of both $\mathrm{PGE}_{2}$ and IL- $1 \beta$ production, as co-production of both in human monocytic assays may predict rabbit pyrogenicity (i.e., fever) in vivo (33). Overall, a common trend was observed. The bacillus Calmette-Guérin (BCG) vaccine, Bexsero and WT dOMV consistently induced the highest production of both $\mathrm{PGE}_{2}$ and IL-1 $\beta . \Delta l p x L I \mathrm{nOMV}$ conversely induced a lower MoDC $\mathrm{PGE}_{2} / \mathrm{IL}-1 \beta$ profile than $\mathrm{WT}$
OMV-based vaccines, suggesting similarity to low reactogenicity pediatric vaccines such as PCV13 and HBV (Figures 5C,D).

\section{$\Delta / p x L I$ nOMV Dissociates Inflammation from Immunogenicity}

Having characterized the relatively low reactogenicity potential of $\Delta l p x L I$ nOMV toward human leukocytes in vitro, we next 


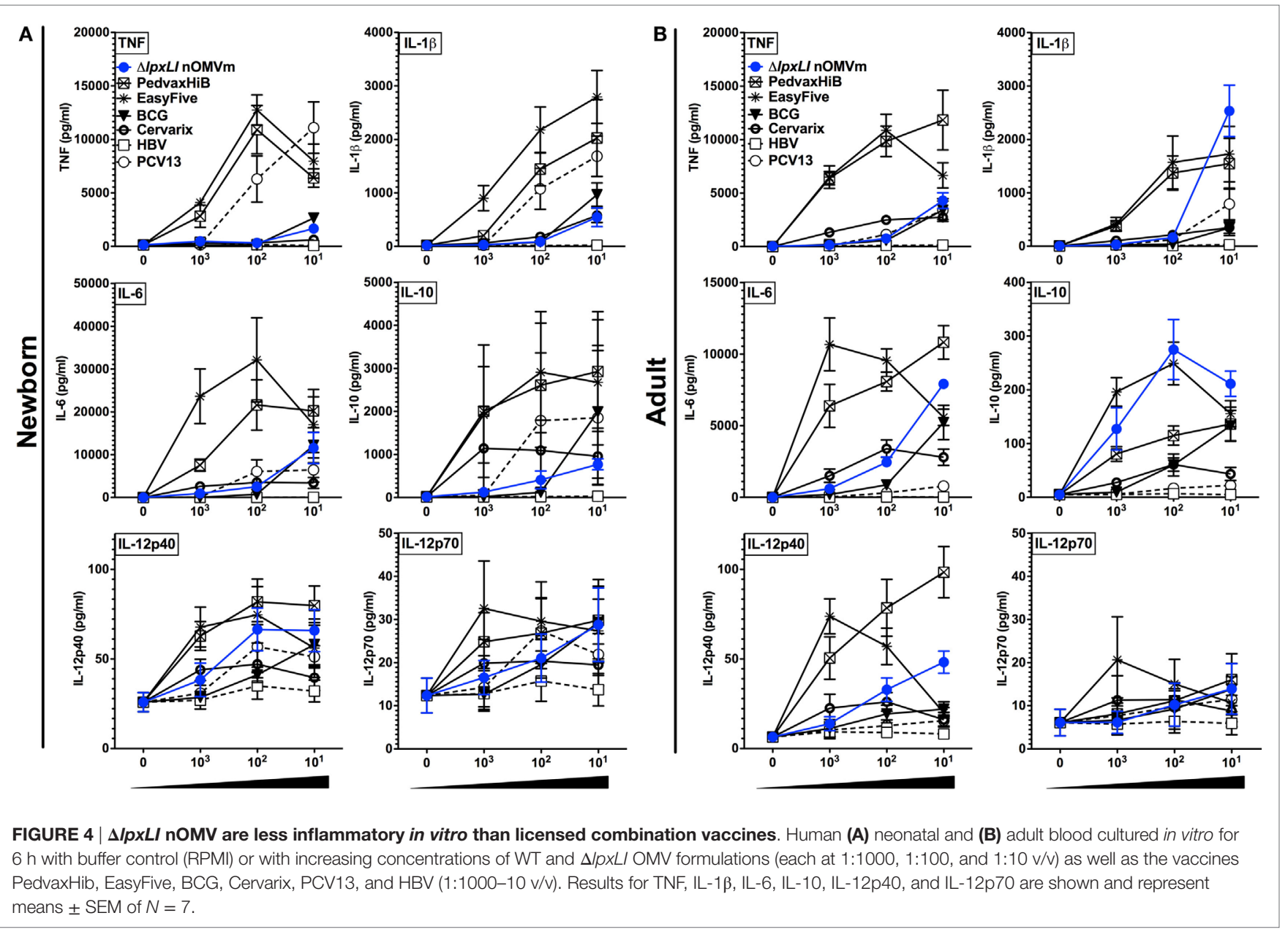

assessed the ability of $\Delta l p x L I$ nOMV to induce immunogenicity in mice in vivo. Four cohorts of 10 mice each (Figure 6) were immunized subcutaneously with Tris/Sucrose (buffer control), $\Delta l p x L I$ nOMV or WT dOMV (2.5 $\mu$ g total protein/dose, dOMVs formulated with Alum), or a 1/10th dose of Bexsero (equivalent to $2.5 \mu \mathrm{g}$ dOMV). Two doses were administered 4 weeks apart, with blood collected 2 weeks after the second dose to obtain serum for SBA. The buffer control alone failed to induce meningococcal bactericidal antibody responses. In marked contrast, Bexsero $(p<0.05)$, WT dOMV $(p<0.01)$, and $\Delta l p x L I$ nOMV $(p<0.01)$ all induced robust and significant antibody responses as compared to baseline (Figure 6). Remarkably, even though $\Delta l p x L I$ nOMVs demonstrated reduced inflammation potential toward human leukocytes in vitro, there was no significant difference in immunogenicity as compared to either Bexsero or WT dOMV.

\section{DISCUSSION}

The majority of global immunization schedules are pediatric, with particular focus on newborns and young infants, yet most vaccine discovery programs do not rationally design vaccine formulations for use in humans in early life. Indeed, even when employed, in vitro modeling of human responses often occurs in late stage vaccine development. Such current pre-clinical approaches may contribute to vaccine formulations inducing sub-optimal responses in the very young (3). To more completely evaluate an OMV-based $N$. meningitidis vaccine, we took a rational vaccine design approach, in which we combined a relevant in vivo model and age-specific human in vitro culture systems that together may better model OMV-induced innate immunomodulatory capacity and immunogenicity potential. Overall, when compared to the WT detergent-extracted OMV (WT dOMV), mutant detergentextracted OMV ( $\Delta l p x L I$ dOMV), and multiple licensed vaccines with respect to innate signaling toward human newborn and adult leukocytes in vitro, a prototype $\Delta l p x L I$ nOMV vaccine generated a pattern of response suggestive of low reactogenicity potential while still demonstrating robust immunogenicity in mice in vivo. Demonstration of non-inferior in vivo immunogenicity of the $\Delta l p x L I$ nOMV vaccine, as compared to Bexsero, is noteworthy, especially as the in vitro DC maturation profiles are divergent. Such a result may indicate that the inclusion of native endotoxin molecules into traditionally designed OMV-based vaccines may not be as essential for immunogenicity as often assumed.

A key concern regarding modern vaccine development is reactogenicity (33), the propensity of a formulation to cause acute inflammatory events either locally, such as erythema or tenderness 


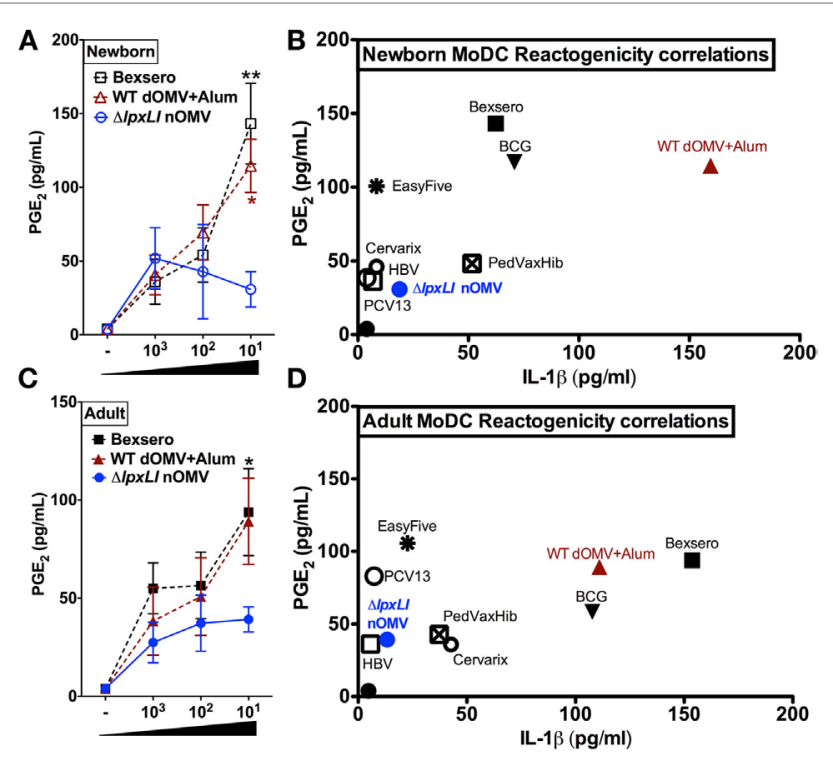

FIGURE $5 \mid \Delta / p x L /$ nOMV-induced lower MoDC-PGE ${ }_{2}$ and -IL-1 $\beta$ than Alum-adjuvanted WT OMV and the licensed Bexsero vaccine. Human newborn and adult MoDCs were cultured in 10\% autologous plasma ( $/ \mathrm{V}$ ) and stimulated for $24 \mathrm{~h}$ with increasing concentrations of OMV vaccines (each at 1:1000, 1:100, and 1:10 v/v). (A) Newborn and (C) adult PGE 2 production, as measured by ELISA. Potential reactogenicity biomarker correlation of $\mathrm{PGE}_{2}$ with $\mathrm{IL}-1 \beta$ for newborn (B) and adult (D) MoDCs after treatment with wild $\mathrm{WT}$ and mutant OMV formulations (at 1:10 v/v) as well as the vaccines PedvaxHib, EasyFive, BCG, Cervarix, PCV13, and HBV (at 1:10 $\mathrm{v} / \mathrm{v})$. Mean $\pm \mathrm{SEM}, n=5-6$. $\mathrm{PGE}_{2}$ levels are depicted with untreated basal levels subtracted. For analyses at individual treatments (e.g., Bexsero 1:10 vs. $\Delta / p x L /$ nOMV 1:10), Wilcoxon test was applied at each concentration and statistical significances are denoted as follows: ${ }^{*} p<0.05$ and ${ }^{* *} p<0.01$.

at the injection site, or systemically, such as fever. In this context, in vitro assays that provide potential reactogenicity biomarker data may be highly advantageous in de-risking formulation selection for in vivo use (21). By employing an in vitro human DC array, we benchmarked the immunomodulatory abilities of an $\Delta l p x L I$ nOMV-based vaccine against several licensed pediatric vaccines. DCs are logical targets for such in vitro studies as they efficiently process antigens for induction of immunity against pathogens and their components (34). The few published studies that assessed the activating effects of OMV on human leukocytes in vitro (35-40) evaluated fixed concentrations with limited cytokine measurements. One study evaluated concentration-dependent OMV responses in human $\mathrm{WB}$ and PBMC assays, solely from adult donors (39). Accordingly, our study is the first to comprehensively investigate (a) the ontogeny of OMV-induced immune responses and (b) the responses of human DCs to OMVs as benchmarked against licensed vaccines. $\triangle l p x L I$ nOMV induced cytokine responses significantly lower than those induced by either Bexsero or licensed vaccines (i.e., PedvaxHIB and EasyFive). As demonstrated by the stronger induction of costimulatory molecule expression by the $\Delta l p x L I$ nOMV with Alum in adult but not newborn MoDCs, the dramatically enhanced human DC IL-1 $\beta$ production in response to Alum-adjuvanted WT dOMV, as well as our prior studies demonstrating distinct ontological effects of

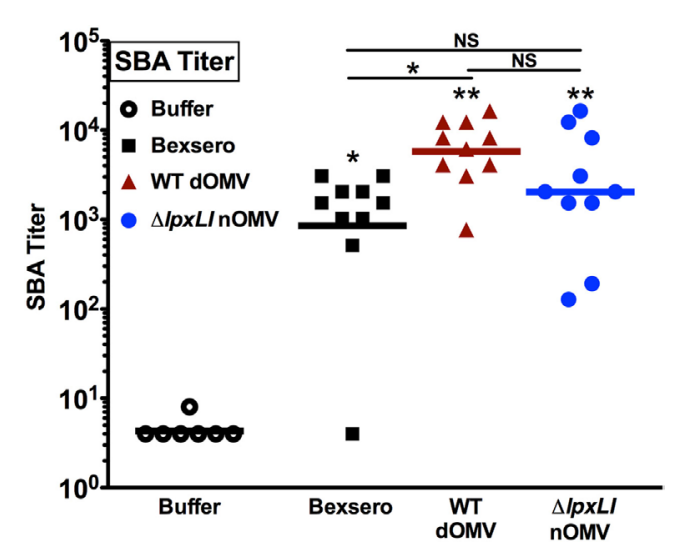

FIGURE $6 \mid \Delta / p x L I$ nOMV induce robust immunogenicity in vivo. Mouse functional antibodies levels were determined by serum bactericidal assays (SBAs) after subcutaneous immunization with $\Delta / p x L /$ nOMV or WT dOMV $(2.5 \mu \mathrm{g}$ total protein/dose) formulations, as well as the vaccines Bexsero (1/10th human dose) or buffer control. Two doses were administered 4 weeks apart, with terminal bleed taken 2 weeks after the second dose. Mutant OMV demonstrated enhanced immunogenicity over buffer control treatment, and equivalent induction of functional antibodies to the WT formulations, $n=10$. Horizontal line indicates geometric mean. Wilcoxon test was applied between buffer and each OMV formulation, or between OMV formulations as indicated. Statistical significance is denoted as follows: ${ }^{*} p<0.05,{ }^{\star *} p<0.01$, and not significant (NS).

Alum on in vitro DC IL-1 $\beta$ production (24), it is reasonable to suggest that our age-specific in vitro systems may be useful tools with respect to characterizing candidate vaccine formulations with or without Alum.

Our study has multiple strengths including (a) robust agespecific human in vitro modeling, (b) extensive benchmarking to multiple licensed vaccines, and (c) confirmation of immunogenicity in vivo. That said, our study also has some limitations including (a) the unavoidable use of different species for in vitro (human) and in vivo (mouse) studies, (b) that in vitro systems, no matter how carefully developed, are imperfect models for responses in vivo, (c) the lack of full concordance in the antigens profile found in Bexsero- which contains added recombinant protein- and our $\triangle l_{p} x L I$ nOMV formulations that do not, and (d) the lack of non-detergent treated-WT strain-derived OMVs that contain WT LPS. While such a formulation was initially not evaluated as part of our current study due to the known cytotoxic effects in humans, based on our results, such WT OMVs may provide a benchmark in future studies employing human in vitro systems. Of note, our experiences to date with in vitro benchmarking suggest that our in vitro studies provide insights relevant in vivo (2). Additionally, studies evaluating OMV vaccine-based reactogenicity in vivo using similar strategies to ours in mice (41) and humans (42) support these conclusions.

Several aspects of our results will prompt future investigations. For example, nucleotide-binding oligomerization domain (NOD)-like receptor-mediated IL-1 $\beta$ production is greater in human newborn cord than adult peripheral blood $(43,44)$ and slowly decreases to adult levels over the first years of life (45). A similar phenomenon for basal $\mathrm{PGE}_{2}$ levels in $\mathrm{WB}$ has been 
observed (32). Therefore, further study of the IL-1 $\beta / \mathrm{PGE}_{2}$ axis as a predictive marker of vaccine reactogenicity may need to take ontogeny into account. Recent use of proteomics (secretomics) has profiled hundreds of proteins released by human monocytes when stimulated in vitro with Alum or TLR agonists, several of which have been validated upon study of licensed adjuvanted vaccines, providing opportunities for further refinement of future adjuvanticity and reactogenicity biomarkers (31). Finally, the evaluation of different pentaacyl lipid A mutants (46) and comparison of their modified agonist properties using age-specific human WB and DC assays should also considered.

The persistently high global burden of meningococcal disease in the very young infants and adolescents provides a compelling rationale for developing additional safe and effective early life vaccines (47). Overall, two key aspects of our study deserve particular emphasis: (a) human in vitro systems model age-specific biomarker responses that may correspond to reactogenicity and immunogenicity as benchmarked to licensed vaccines and (b) these in vitro systems may enable systematic comparison of formulations with and without candidate adjuvants early in the design process to inform translational development. Overall, if further validated, such an approach of in vitro assay systeminformed age-specific vaccine development may open new paths to more precise vaccine development for distinct vulnerable populations.

\section{AUTHOR CONTRIBUTIONS}

DD, GD, and OL designed the study. DD, WC, SJ, SB, IB, CP, and $\mathrm{SH}$ conducted the in vitro experiments. $\mathrm{HS}, \mathrm{SA}$, and JF produced $\mathrm{OMV}$ vaccines and conducted the in vivo experiments. DD wrote the manuscript. OL provided overall mentorship and assisted in writing the manuscript. HS, WC, SJ, SB, IB, CP, SH, SA, JF, and GD contributed to helpful discussions and the careful approval of the final manuscript. All the authors have given final approval for the version submitted for publication.

\section{REFERENCES}

1. Prabhudas M, Adkins B, Gans H, King C, Levy O, Ramilo O, et al. Challenges in infant immunity: implications for responses to infection and vaccines. Nat Immunol (2011) 12:189-94. doi:10.1038/ni0311-189

2. Dowling DJ, Levy O. Ontogeny of early life immunity. Trends Immunol (2014) 35:299-310. doi:10.1016/j.it.2014.04.007

3. Dowling DJ, Levy O. Pediatric vaccine adjuvants: components of the modern vaccinologist's toolbox. Pediatr Infect Dis J (2015) 34:1395-8. doi:10.1097/ INF.0000000000000893

4. Plotkin SA, Orenstein WA, Offit PA. Vaccines. Sixth ed. Philadelphia, PA: Saunders (2012).

5. Ladhani SN, Ramsay M, Borrow R, Riordan A, Watson JM, Pollard AJ. Enter $\mathrm{B}$ and $\mathrm{W}$ : two new meningococcal vaccine programmes launched. Arch Dis Child (2016) 101:91-5. doi:10.1136/archdischild-2015-308928

6. Andrews SM, Pollard AJ. A vaccine against serogroup B Neisseria meningitidis: dealing with uncertainty. Lancet Infect Dis (2014) 14:426-34. doi:10.1016/ S1473-3099(13)70341-4

7. Sanders H, Kaaijk P, Van Den Dobbelsteen GP. Preclinical evaluation of MenB vaccines: prerequisites for clinical development. Expert Rev Vaccines (2013) 12:31-42. doi:10.1586/erv.12.137

\section{ACKNOWLEDGMENTS}

The authors thank the members of the OL's Laboratory for assistance with phlebotomy as well as helpful discussions. The authors thank their donors and acknowledge the assistance of the Labor and Delivery staff at both The Brigham and Women's Hospital, Boston and Beth Israel Deaconess Medical Center, Boston. The authors are grateful for the mentorship and support of Drs. Michael Wessels and Gary R. Fleisher.

\section{FUNDING}

This study was supported by Janssen Vaccines and Prevention B.V. (formerly Crucell Holland B.V.). OL's laboratory is supported by U.S. National Institutes of Health (NIH) grants 1R01AI100135-01, and 3R01AI067353-05S1, the National Institutes of Allergy and Infectious Diseases (NIAID), NIH, Department of Health and Human Services, NIH UO1 award Molecular Mechanisms of Combination Adjuvants (1U01AI124284-01), Adjuvant Discovery Program Contract No. HHSN272201400052C as well as Global Health (OPPGH5284) and Grand Challenges Explorations (OPP1035192) awards from the Bill \& Melinda Gates Foundation. CP was supported by the scholarship "J. Miglierina," Fondazione Comunitaria del Varesotto, Varese, Italy. OL's Laboratory has received sponsored research support from VentiRx Pharmaceuticals, 3M Drug Delivery Systems, MedImmune, Crucell (Johnson \& Johnson), Shire, and an internal Boston Children's Hospital award to the Precision Vaccines Program.

\section{SUPPLEMENTARY MATERIAL}

The Supplementary Material for this article can be found online at http://journal.frontiersin.org/article/10.3389/fimmu.2016.00562/ full\#supplementary-material.

8. Serruto D, Bottomley MJ, Ram S, Giuliani MM, Rappuoli R. The new multicomponent vaccine against meningococcal serogroup B, 4CMenB: immunological, functional and structural characterization of the antigens. Vaccine (2012) 30(Suppl 2):B87-97. doi:10.1016/j.vaccine.2012.01.033

9. Fredriksen JH, Rosenqvist E, Wedege E, Bryn K, Bjune G, Froholm LO, et al. Production, characterization and control of MenB-vaccine "Folkehelsa": an outer membrane vesicle vaccine against group B meningococcal disease. NIPH Ann (1991) 14:67-79; discussion 79-80.

10. CHMP. Annex I - Summary of Product Characteristics. European Medicines Agency European Public Assessment Report (EPAR). Bexsero meningococcal group-B vaccine, EMEA/H/C/002333, London (2015).

11. Tenenbaum T, Niessen J, Schroten $H$. Severe upper extremity dysfunction after 4CMenB vaccination in a young infant. Pediatr Infect Dis J (2016) 35:94-6. doi:10.1097/INF.0000000000000917

12. Vesikari T, Esposito S, Prymula R, Ypma E, Kohl I, Toneatto D, et al Immunogenicity and safety of an investigational multicomponent, recombinant, meningococcal serogroup B vaccine (4CMenB) administered concomitantly with routine infant and child vaccinations: results of two randomised trials. Lancet (2013) 381:825-35. doi:10.1016/S0140-6736(12)61961-8

13. Prymula R, Esposito S, Zuccotti GV, Xie F, Toneatto D, Kohl I, et al. A phase 2 randomized controlled trial of a multicomponent meningococcal serogroup 
B vaccine (I). Hum Vaccin Immunother (2014) 10:1993-2004. doi:10.4161/ hv. 28666

14. Vipond C, Findlay L, Feavers I, Care R. Limitations of the rabbit pyrogen test for assessing meningococcal OMV based vaccines. ALTEX (2016) 33:47-53. doi:10.14573/altex.1509291

15. Van Der Ley P, Steeghs L, Hamstra HJ, Ten Hove J, Zomer B, Van Alphen L. Modification of lipid A biosynthesis in Neisseria meningitidis lpxL mutants: influence on lipopolysaccharide structure, toxicity, and adjuvant activity. Infect Immun (2001) 69:5981-90. doi:10.1128/IAI.69.10.59815990.2001

16. Van De Waterbeemd B, Streefland M, Van Der Ley P, Zomer B, Van Dijken H, Martens D, et al. Improved OMV vaccine against Neisseria meningitidis using genetically engineered strains and a detergent-free purification process. Vaccine (2010) 28:4810-6. doi:10.1016/j.vaccine.2010. 04.082

17. Dowling D, Hamilton CM, O’Neill SM. A comparative analysis of cytokine responses, cell surface marker expression and MAPKs in DCs matured with LPS compared with a panel of TLR ligands. Cytokine (2008) 41:254-62. doi:10.1016/j.cyto.2007.11.020

18. Steeghs L, Van Vliet SJ, Uronen-Hansson H, Van Mourik A, Engering A, Sanchez-Hernandez M, et al. Neisseria meningitidis expressing lgtB lipopolysaccharide targets DC-SIGN and modulates dendritic cell function. Cell Microbiol (2006) 8:316-25. doi:10.1111/j.1462-5822.2005.00623.x

19. Pettengill MA, Van Haren SD, Levy O. Soluble mediators regulating immunity in early life. Front Immunol (2014) 5:457. doi:10.3389/fimmu.2014. 00457

20. Levitz SM, Golenbock DT. Beyond empiricism: informing vaccine development through innate immunity research. Cell (2012) 148:1284-92. doi:10.1016/j.cell.2012.02.012

21. Mastelic B, Garcon N, Del Giudice G, Golding H, Gruber M, Neels P, et al. Predictive markers of safety and immunogenicity of adjuvanted vaccines. Biologicals (2013) 41:458-68. doi:10.1016/j.biologicals.2013.08.006

22. Lewis DJ, Lythgoe MP. Application of "systems vaccinology" to evaluate inflammation and reactogenicity of adjuvanted preventative vaccines. J Immunol Res (2015) 2015:909406. doi:10.1155/2015/909406

23. Dowling DJ, Tan Z, Prokopowicz ZM, Palmer CD, Matthews MA, Dietsch GN, et al. The ultra-potent and selective TLR8 agonist VTX-294 activates human newborn and adult leukocytes. PLoS One (2013) 8:e58164. doi:10.1371/ journal.pone.0058164

24. Philbin VJ, Dowling DJ, Gallington LC, Cortes G, Tan Z, Suter EE, et al. Imidazoquinoline toll-like receptor 8 agonists activate human newborn monocytes and dendritic cells through adenosine-refractory and caspase1-dependent pathways. J Allergy Clin Immunol (2012) 130(195-204):e199. doi:10.1016/j.jaci.2012.02.042

25. Palmer CD, Ninkovic J, Prokopowicz ZM, Mancuso CJ, Marin A, Andrianov $\mathrm{AK}$, et al. The effect of stable macromolecular complexes of ionic polyphosphazene on HIV Gag antigen and on activation of human dendritic cells and presentation to T-cells. Biomaterials (2014) 35:8876-86. doi:10.1016/j. biomaterials.2014.06.043

26. Ganapathi L, Van Haren S, Dowling DJ, Bergelson I, Shukla NM, Malladi SS, et al. The imidazoquinoline toll-like receptor-7/8 agonist hybrid-2 potently induces cytokine production by human newborn and adult leukocytes. PLoS One (2015) 10:e0134640. doi:10.1371/journal.pone.0134640

27. Van De Waterbeemd B, Zomer G, Kaaijk P, Ruiterkamp N, Wijffels RH, Van Den Dobbelsteen GP, et al. Improved production process for native outer membrane vesicle vaccine against Neisseria meningitidis. PLoS One (2013) 8:e65157. doi:10.1371/journal.pone.0065157

28. Sanders H. Investigation of the Potential of PorA and FetA as Meningococcal Vaccine Components. Oxford: University of Oxford (2012).

29. Norheim G, Aase A, Caugant DA, Hoiby EA, Fritzsonn E, Tangen T, et al. Development and characterisation of outer membrane vesicle vaccines against serogroup A Neisseria meningitidis. Vaccine (2005) 23:3762-74. doi:10.1016/j. vaccine.2005.02.021

30. Maslanka SE, Gheesling LL, Libutti DE, Donaldson KB, Harakeh HS, Dykes JK, et al. Standardization and a multilaboratory comparison of Neisseria meningitidis serogroup A and C serum bactericidal assays. The Multilaboratory Study Group. Clin Diagn Lab Immunol (1997) 4:156-67.
31. Oh DY, Dowling DJ, Ahmed S, Choi H, Brightman S, Bergelson I, et al. Adjuvant-induced human monocyte secretome profiles reveal adjuvant- and age-specific protein signatures. Mol Cell Proteomics (2016) 15(6):1877-94. doi:10.1074/mcp.M115.055541

32. Van Haren SD, Ganapathi L, Bergelson I, Dowling DJ, Banks M, Samuels $\mathrm{RC}$, et al. In vitro cytokine induction by TLR-activating vaccine adjuvants in human blood varies by age and adjuvant. Cytokine (2016) 83:99-109. doi:10.1016/j.cyto.2016.04.001

33. Zaitseva M, Romantseva T, Blinova K, Beren J, Sirota L, Drane D, et al. Use of human MonoMac6 cells for development of in vitro assay predictive of adjuvant safety in vivo. Vaccine (2012) 30:4859-65. doi:10.1016/j.vaccine. 2012.05.002

34. Bachmann MF, Jennings GT. Vaccine delivery: a matter of size, geometry, kinetics and molecular patterns. Nat Rev Immunol (2010) 10:787-96. doi:10.1038/nri2868

35. Koeberling O, Seubert A, Granoff DM. Bactericidal antibody responses elicited by a meningococcal outer membrane vesicle vaccine with overexpressed factor $\mathrm{H}$-binding protein and genetically attenuated endotoxin. J Infect Dis (2008) 198:262-70. doi:10.1086/589308

36. Steeghs L, Keestra AM, Van Mourik A, Uronen-Hansson H, Van Der Ley P, Callard R, et al. Differential activation of human and mouse toll-like receptor 4 by the adjuvant candidate LpxL1 of Neisseria meningitidis. Infect Immun (2008) 76:3801-7. doi:10.1128/IAI.00005-08

37. Koeberling O, Giuntini S, Seubert A, Granoff DM. Meningococcal outer membrane vesicle vaccines derived from mutant strains engineered to express factor $\mathrm{H}$ binding proteins from antigenic variant groups 1 and 2. Clin Vaccine Immunol (2009) 16:156-62. doi:10.1128/CVI.00403-08

38. Fransen F, Hamstra HJ, Boog CJ, Van Putten JP, Van Den Dobbelsteen GP, Van Der Ley P. The structure of Neisseria meningitidis lipid A determines outcome in experimental meningococcal disease. Infect Immun (2010) 78:3177-86. doi:10.1128/IAI.01311-09

39. Stoddard MB, Pinto V, Keiser PB, Zollinger W. Evaluation of a whole-blood cytokine release assay for use in measuring endotoxin activity of group $B$ Neisseria meningitidis vaccines made from lipid A acylation mutants. Clin Vaccine Immunol (2010) 17:98-107. doi:10.1128/CVI.00342-09

40. Koeberling O, Seubert A, Santos G, Colaprico A, Ugozzoli M, Donnelly J, et al. Immunogenicity of a meningococcal native outer membrane vesicle vaccine with attenuated endotoxin and over-expressed factor $\mathrm{H}$ binding protein in infant rhesus monkeys. Vaccine (2011) 29:4728-34. doi:10.1016/j. vaccine.2011.04.095

41. Daniels-Treffandier H, De Nie K, Marsay L, Dold C, Sadarangani M, ReyesSandoval A, et al. Impact of reducing complement inhibitor binding on the immunogenicity of native Neisseria meningitidis outer membrane vesicles. PLoS One (2016) 11:e0148840. doi:10.1371/journal.pone.0148840

42. Keiser PB, Biggs-Cicatelli S, Moran EE, Schmiel DH, Pinto VB, Burden RE, et al. A phase 1 study of a meningococcal native outer membrane vesicle vaccine made from a group B strain with deleted lpxL1 and synX, over-expressed factor $\mathrm{H}$ binding protein, two PorAs and stabilized OpcA expression. Vaccine (2011) 29:1413-20. doi:10.1016/j.vaccine.2010. 12.039

43. Kollmann TR, Crabtree J, Rein-Weston A, Blimkie D, Thommai F, Wang $\mathrm{XY}$, et al. Neonatal innate TLR-mediated responses are distinct from those of adults. JImmunol (2009) 183:7150-60. doi:10.4049/jimmunol. 0901481

44. Reikie BA, Adams RC, Ruck CE, Ho K, Leligdowicz A, Pillay S, et al. Ontogeny of toll-like receptor mediated cytokine responses of South African infants throughout the first year of life. PLoS One (2012) 7:e44763. doi:10.1371/ journal.pone. 0044763

45. Burl S, Townend J, Njie-Jobe J, Cox M, Adetifa UJ, Touray E, et al. Agedependent maturation of toll-like receptor-mediated cytokine responses in Gambian infants. PLoS One (2011) 6:e18185. doi:10.1371/journal. pone. 0018185

46. Pupo E, Hamstra HJ, Meiring H, Van Der Ley P. Lipopolysaccharide engineering in Neisseria meningitidis: structural analysis of different pentaacyl lipid A mutants and comparison of their modified agonist properties. J Biol Chem (2014) 289:8668-80. doi:10.1074/jbc.M114.554345

47. Liu L, Johnson HL, Cousens S, Perin J, Scott S, Lawn JE, et al. Global, regional, and national causes of child mortality: an updated systematic analysis for 
2010 with time trends since 2000. Lancet (2012) 379:2151-61. doi:10.1016/ S0140-6736(12)60560-1

Conflict of Interest Statement: HS, SA, JF, and GD are employees of Janssen Vaccines and Prevention B.V., part of the Janssen pharmaceutical companies of Johnson \& Johnson. This study was funded by Janssen Vaccines and Prevention B.V. (formerly Crucell B.V.). All the other authors report no potential conflicts.
Copyright $\odot 2016$ Dowling, Sanders, Cheng, Joshi, Brightman, Bergelson, Pietrasanta, van Haren, van Amsterdam, Fernandez, van den Dobbelsteen and Levy. This is an open-access article distributed under the terms of the Creative Commons Attribution License (CC BY). The use, distribution or reproduction in other forums is permitted, provided the original author(s) or licensor are credited and that the original publication in this journal is cited, in accordance with accepted academic practice. No use, distribution or reproduction is permitted which does not comply with these terms. 